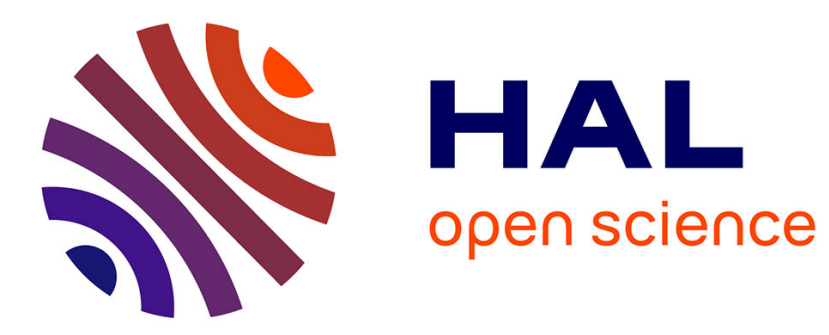

\title{
La territorialisation des politiques scolaires: l'exemple des collèges du bassin de Gap
}

\author{
Patrice Caro, Emmanuel Faivre, Frédéric Grosjean
}

\section{To cite this version:}

Patrice Caro, Emmanuel Faivre, Frédéric Grosjean. La territorialisation des politiques scolaires: l'exemple des collèges du bassin de Gap. Annales de géographie, 2006, 650, pp.434-448. halshs00840459

\section{HAL Id: halshs-00840459 \\ https://shs.hal.science/halshs-00840459}

Submitted on 2 Jul 2013

HAL is a multi-disciplinary open access archive for the deposit and dissemination of scientific research documents, whether they are published or not. The documents may come from teaching and research institutions in France or abroad, or from public or private research centers.
L'archive ouverte pluridisciplinaire HAL, est destinée au dépôt et à la diffusion de documents scientifiques de niveau recherche, publiés ou non, émanant des établissements d'enseignement et de recherche français ou étrangers, des laboratoires publics ou privés. 


\title{
La territorialisation des politiques scolaires : l'exemple des collèges du bassin de Gap
}

\section{School policies' territorialisation: the example of junior high schools in the Gap basin}

\author{
Caro P. \\ Maître de Conférence en géographie, Laboratoire ThéMA UMR 6049 CNRS, Université \\ de Franche-Comté.
}

\section{Faivre E.}

Docteur en géographie, Membre du laboratoire ThéMA UMR 6049 CNRS, Université de Franche-Comté.

\section{Grosjean F.}

Docteur en géographie, Membre du laboratoire ThéMA UMR 6049 CNRS, Université de Franche-Comté.

Résumé Le bassin de Gap connaît un accroissement démographique en milieu périurbain, ce qui nécessite l'analyse de la carte scolaire afin de créer de nouveaux collèges. Ce bassin de population, marqué par des contraintes fortes, est un véritable espace vécu dans le sens où il est structuré par des aires de recrutement résultant des pratiques spatiales des élèves et de leurs familles. Une étude approfondie des structures et des dynamiques spatiales de ce bassin permet de tirer quelques leçons quant au fonctionnement d'un territoire scolaire.

Abstract The Gap area is experiencing a population growth in periurban zones, which requires the analysis of the school map in order to create new secondary schools. This population basin, marked by strong constraints, proves to be a true lived space: it is structured by recruitment areas stemming from spatial practices of school children and their families. A thorough study of the structures and spatial dynamics of this basin allows us to draw some lessons about the functioning of a school territory.

Mots clés Politiques scolaires, collèges, bassin de Gap, zones périurbaines, aires de recrutement.

Key words School policies, secondary school, Gap area, periurban zones, recruitement areas.

\section{Introduction ${ }^{1}$}

Les évolutions territoriales et démographiques à l'œuvre depuis une vingtaine d'années en France (périurbanisation, rurbanisation, explosion des navettes...) impliquent une nouvelle approche des processus de scolarisation. Elles induisent des changements dans l'organisation des territoires scolaires autour des aires urbaines françaises en croissance démographique. 
Quand le peuplement change, la carte scolaire doit évoluer. Les espaces en croissance démographique deviennent vite sous-équipés en établissements scolaires, en termes de capacités d'accueil et de répartition spatiale. Tel est le cas du bassin de formation de Gap où une forte croissance démographique en milieu périurbain appelle la création de nouveaux collèges.

Mais la création de nouveaux établissements modifie profondément le territoire scolaire existant. Pour réfléchir par exemple à la localisation de nouveaux collèges, il faut étudier le fonctionnement et les caractéristiques de ce territoire scolaire. En effet, au-delà de l'apparente uniformité des 6900 collèges localisés en métropole ${ }^{2}$ (programmes nationaux par exemple), il existe une vraie diversité des territoires scolaires, comme l'a montré M. Moracchini au sujet des collèges des montagnes d'Auvergne (Moracchini C., 1992). Le collège introduit donc de la discontinuité dans l'espace (François J.C., 1998). La territorialisation de la politique scolaire (Giband D., Holz J.-M., 2001) et la compréhension du fonctionnement du territoire scolaire doivent prendre en compte cette diversité et ces discontinuités.

Dans le cas du territoire scolaire actuel de Gap, toutes ces dimensions sous-tendent l'interrogation sur la modification de la carte de l'offre de formation initiale, en lien avec la croissance démographique du milieu périurbain. Cet article met en évidence le fonctionnement du territoire scolaire que constitue le bassin de formation de Gap. Il insiste sur les principes présidant à la délimitation des aires de recrutements des collèges et les pratiques spatiales des habitants concernés par ce service, leur ressenti. La notion d'espace vécu prend ici tout son sens avec l'étude du comportement et des représentations des habitants d'une part, et des élus concernés, d'autre part.

Après avoir présenté les caractères du territoire scolaire correspondant au bassin de formation de Gap en milieu montagnard, on verra que les aires de recrutement des collèges sont conformes à la carte scolaire, en raison des pratiques spatiales des habitants. Puis, on mesurera le ressenti des familles et des élèves en matière de scolarisation et de vie au collège. De manière plus générale, les difficultés à créer ou modifier un territoire scolaire ne s'expliquent-elles pas en partie par les représentations que certains acteurs se font du territoire ? L'analyse d'un territoire scolaire s'effectue avant tout, pour certaines personnes, au moyen d'images et de stéréotypes. Il en résulte une connaissance partielle, et parfois même erronée, des réalités territoriales

1 Résultats issus d'une étude menée par le laboratoire ThéMA pour le Conseil Général des HautesAlpes concernant la recherche d'implantation de futur(s) collège(s) dans le bassin de formation de Gap visant à faire face à une situation de sureffectif futur, étude menée de Septembre 2004 à Janvier 2005 utilisant trois sources de données complémentaires : la Base Elèves Académique (BEA) gérée par le Rectorat d'Aix-Marseille, une enquête réalisée par nos soins auprès de 1101 collégiens et 704 écoliers du territoire d'étude (taux de réponse respectifs : $58 \%$ et $63 \%$ ) et des entretiens auprès des acteurs, concernés directement ou indirectement (maires, inspecteur d'académie, inspecteurs de l'Education nationale, représentants des professeurs et des parents d'élèves, chefs d'établissement, agence de développement, etc.).

2 Voir à ce sujet l'article intitulé "La diversité des lieux d'enseignement », in Education et formations $n^{\circ} 66$ de juillet-décembre 2003, MEN. 
des processus scolaires (exemple de distances-temps de déplacement exagérées ou minorées par rapport à la réalité). Les élèves eux-mêmes procèdent de la sorte et choisissent un établissement scolaire en fonction de leur représentation de celui-ci (Sittler M., 1998).

\section{Le territoire scolaire gapencais}

Le bassin de formation de Gap s'inscrit dans le département rural des Hautes-Alpes (121 000 habitants en 1999, 22 habitants au km², $5549 \mathrm{~km}^{2}$ ) en milieu montagnard peu peuplé. Il réunit 126 communes réparties sur 17 cantons, compte 20 communautés de communes et un pays. Avec $18 \%$ de jeunes âgés de moins de 15 ans, le département des Hautes-Alpes compte parmi les départements jeunes de France métropolitaine, sous l'effet d'un solde naturel et d'un solde migratoire positifs.

\subsection{Un territoire scolaire marqué par des contraintes fortes}

Le bassin de formation de Gap, espace montagneux, est soumis à des contraintes fortes de différentes natures. Un fort enclavement et un climat à l'ensoleillement exceptionnel, aux conditions climatiques assez difficiles en période hivernale, posent de sérieux problèmes aux transports scolaires et à la circulation routière en général (temps de transport scolaire parfois très importants, insécurité hivernale). Les principaux axes de transport, notamment routiers, respectent les vallées et les cols. Ces contraintes influencent également grandement les formes d'habitat. Le peuplement du bassin se compose en effet d'une alternance d'espaces polarisés par de gros bourgs centres et d'autres parsemés de hameaux dispersés. Le territoire scolaire de Gap offre un visage rural affirmé, puisque aucune commune ne compte plus de 3500 habitants (hormis Gap).

L'armature urbaine du bassin de formation est structurée par le pôle urbain de Gap qui concentre environ 40000 habitants, soit un tiers de la population du département et plus de la moitié de la population du bassin. La population totale de ce dernier, maillé par huit collèges, s'élève à 80000 habitants environ, soit un peu moins que la population totale de l'arrondissement de Gap. L'aire urbaine de Gap comptait 44700 habitants en 1999 (150 aire urbaine de France métropolitaine, accroissement démographique dans la logique du développement antérieur ${ }^{3}$ ), soit légèrement plus que la commune de Gap elle-même (38 600 habitants en 1999). Par conséquent, on ne peut pas parler de bassin polarisé par une grande agglomération gapençaise, d'autant plus que les «bourgs centres » (Veynes, Saint-Bonnet en Champsaur, Tallard, Chorges) forment davantage des pôles périphériques distants, qu'un tissu périurbain continu. Les espaces périurbains sont situés à un quart d'heure de voiture de Gap, que ce soit

3 Julien P., 2000, Recensement de 1999, poursuite d'une urbanisation très localisée. INSEE première $n^{\circ} 692$, page 3 . 


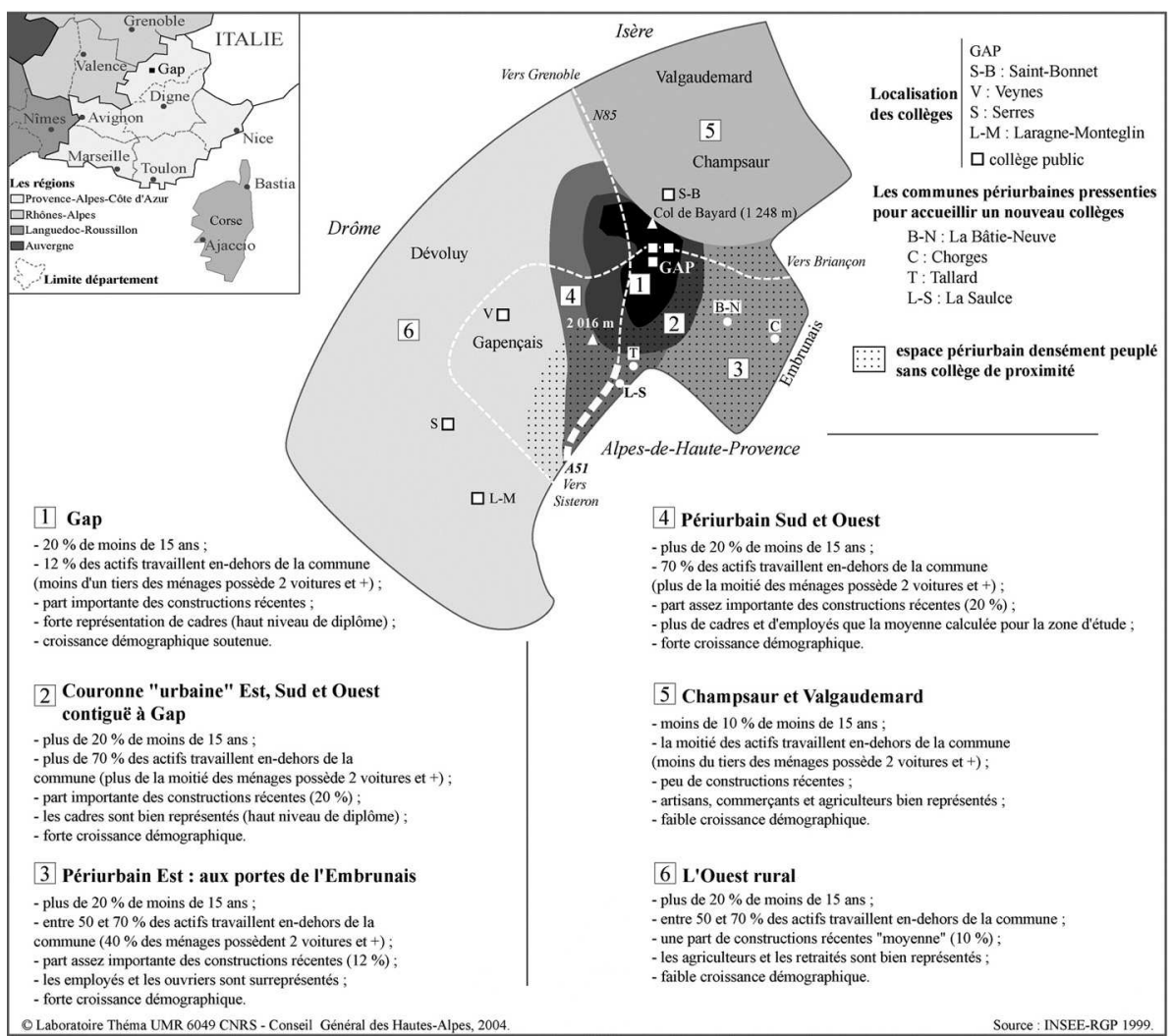

Fig 1 Le bassin de formation de Gap et ses collèges.

The educational Gap basin and its junior high schools.

à l'est (Chorges/La Bâtie Neuve : environ 3500 habitants en 1999), ou à l'ouest (Tallard/La Saulce : 2200 habitants en 1999) de Gap.

Ce bassin de formation connaît une croissance démographique supérieure aux moyennes nationales et régionales. La population du bassin s'est accru de $8 \%$ entre 1990 et 1999 , contre $7 \%$ pour le département des Hautes-Alpes, et $3 \%$ au plan national. Ce gain résulte en grande partie des flux migratoires entrants ( 1 point de solde naturel -naissances- et 7 points de flux migratoires) ${ }^{4}$. Ce dynamisme bénéficie en premier lieu aux espaces périurbains de l'est et du sud de Gap. Ces espaces profitent du desserrement de Gap et d'une intensification de la pression foncière survenue ces dernières années (notamment dans les espaces périurbains situés à l'est et ouest de Gap). À l'avenir, cette situation démographique semble confirmée, puisque les projections tendancielles pour la zone d'emploi de Gap ${ }^{5}$ pré-

4 Sources INSEE - RGP 99.

5 Sources : INSEE - RGP 1999 - projections OMPHALE. 
voient une hausse de 6 à $7 \%$ entre 1999 et 2008, et de 5 à $6 \%$ entre 2009 et 2017. Les espaces périurbains risquent donc de se trouver en situation de sous équipement scolaire avec ce processus de croissance démographique et de desserrement urbain.

Les habitudes de vie des populations du bassin de formation, leurs pratiques spatiales ${ }^{6}$, induisent un découpage de ce territoire scolaire en six sous-ensembles (figure 1). La structure spatiale du bassin se résume donc au modèle centre-périphérie. Plusieurs couronnes d'une dizaine de communes ceinturent partiellement le coeur constitué par l'aire urbaine de Gap. Cette configuration est sans doute assez représentative de l'organisation spatiale des petites aires urbaines françaises en croissance démographique et leurs marges.

\subsection{Un besoin de collèges supplémentaires dans ce bassin de formation}

En 2004-2005, le bassin de formation de Gap défini par l'Inspection Académique compte sept collèges publics réunissant 3774 élèves, et un collège privé de 510 élèves. On distingue deux groupes d'établissements. Les trois collèges "urbains" de Mauzan, Centre et Fontreyne comptent chacun plus de 800 élèves. À l'inverse, les quatre collèges ruraux de Laragne, Veynes, Saint-Bonnet et Serres apparaissent comme des établissements de très petite taille (moins de 200 élèves). La taille moyenne des collèges publics du bassin de formation gapençais (hors collège privé) s'élève donc à 539 élèves, contre 417 élèves en moyenne au plan national (collège public en 2003). ${ }^{7}$

Depuis 1999, les collèges urbains connaissent un accroissement de leurs inscriptions, sauf depuis 2003 où une certaine stabilité, voire une légère érosion des effectifs, s'est engagée. De leur côté, les collèges ruraux sont marqués par une relative stabilité du nombre d'élèves. À l'avenir, les effectifs devraient fluctuer entre 2300 et 2500 collégiens (hors SEGPA) au sein des trois collèges de Gap, sans que ces derniers ne soient saturés (600-700 élèves dans le collège Fontreyne, 900 dans les collèges Mauzan et Centre). Mais une augmentation brutale en 2012-2013 porterait le nombre de collégiens scolarisés au sein des trois établissements de Gap à $2700^{8}$, soit une moyenne de 900 élèves par entité, conduisant à une situation de sureffectif manifeste.

Les relations du bassin de formation de Gap avec les autres bassins sont ténues puisque seuls 12 collégiens résidant au sein du bassin de formation gapençais «sortent » de ce dernier pour aller étudier au collège d'Embrun (11) et au collège Vauban de Briançon (1). À l'inverse, les collèges du bassin de formation gapençais attirent 97 élèves extérieurs, dont 67 jeunes drômois liés

6 Les indicateurs utilisés sont issus du RGP 1999 INSEE : démographie (dynamique pop 90-99, part des jeunes de moins de 15 ans), niveau social (niveau de diplôme 99, PCS 99), indice de mobilité (part des actifs ne travaillant pas dans leur commune de référence, part des ménages possédant 2 voitures et plus), foncier (part des logements construit en 1999, accès à la propriété).

7 Source Ministère de l'Éducation Nationale.

8 Source Inspection Académique des Hautes-Alpes, confirmé par les projections de l'étude du laboratoire ThéMA. 


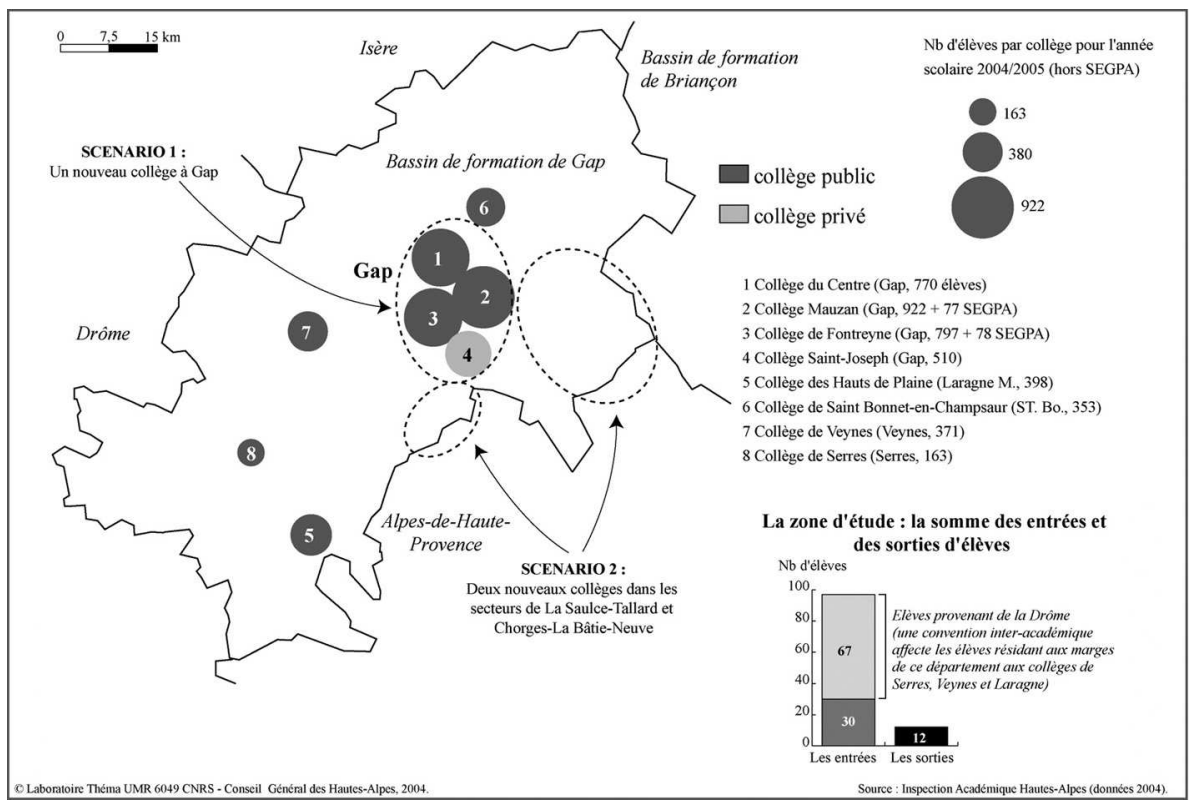

Fig 2 Nombre de collèges et entrées-sorties du bassin de formation de Gap. Numbers of junior high school and entries/departures of educational Gap basin.

par une convention inter-académique. Au total, les mouvements avec d'autres bassins de formation sont donc très réduits puisque seulement 12 élèves " sortent » et 30 élèves «entrent» (hors convention avec la Drôme) sur environ 3800 collégiens, soit seulement $1 \%$ du total des collègiens du bassin.

Afin de mieux cerner l'incidence de ces caractères du bassin de formation sur le comportement des ménages et des élèves, il convient d'observer les pratiques spatiales des habitants entre lieu de résidence et de travail, d'étude, au travers des aires de recrutement des collèges. Ces pratiques spatiales quotidiennes font véritablement le territoire scolaire (appropriation, sentiment d'appartenance à un collège...). L'implantation de nouveaux collèges doit tenir compte de ces pratiques spatiales, elle doit donc se faire en connaissant bien le territoire scolaire de Gap. Par conséquent, dans quelles mesures ces caractéristiques territoriales influencent-elles la vie des collégiens, notamment le choix du collège et le temps pour s'y rendre ?

\section{Des aires de recrutement conformes à la carte scolaire}

Chacun des sept collèges publics est rattaché à un secteur de recrutement de ses effectifs. Mieux connu sous l'appellation de «carte scolaire », 9 ce décou-

9 Ce document cadre passe sous l'autorité des Conseils généraux à partir du 01/01/2005. 
page respecte très souvent le découpage cantonal. Les aires de recrutement des collèges apparaissent assez largement conformes à la carte scolaire. Fautil y voir l'effet de pratiques spatiales très attentives à l'accessibilité et aux distances-temps? Est-ce le résultat d'une certaine discipline des parents d'élèves face à la carte scolaire, un recours très faible aux dérogations ?

\subsection{Des aires de recrutement résultant des pratiques spatiales}

La carte scolaire est assez bien respectée puisque la quasi totalité des communes comprises au sein d'un secteur de rattachement envoient au moins $70 \%$ de leurs enfants dans le collège prévu. Mais des différences existent selon le type de collège.

Ainsi, les quatre collèges ruraux, tel que celui de Saint-Bonnet, recrutent très peu en dehors de leur secteur "théorique ». En revanche, les trois collèges urbains recrutent plus largement au-delà de leur secteur " théorique ». Par exemple, le collège Centre présente l'aire de recrutement la plus large avec plus de $7 \%$ de ses élèves résidant hors de Gap, c'est-à-dire en dehors de son secteur "théorique » de recrutement. Il constitue le collège le plus attractif, même si ce mouvement ne concerne que 46 élèves sur 770. L'aire de recrutement du collège privé Saint-Joseph témoigne d'une autre logique spatiale, avec un champ d'action très élargi, passant outre les règles de l'organisation territoriale du service public.

\subsection{Peu de dérogations à la carte scolaire}

La superposition des aires de recrutement avec la carte scolaire, c'est à dire les périmètres des secteurs «théoriques » de l'Inspection Académique fait apparaître quelques écarts. Les données de l'Inspection académique, ainsi que notre enquête par questionnaire, montrent que la part des dérogations avoisine $10 \%$ des effectifs collégiens du bassin de formation gapençais. Là aussi, deux grands types de collèges coexistent. D'une part, on distingue «les attractifs » qui comptent plus de dérogations pour entrer que de dérogations pour sortir (collèges Centre, de Veynes, de Laragne et de Serres). D'autre part, "les répulsifs » réunissant davantage de dérogations pour sortir que de dérogations pour entrer (collèges Fontreyne, Mauzan et de Saint-Bonnet). Au total, le collège Centre fait figure de collège le plus attractif et celui de Fontreyne le plus répulsif. Il est à noter que deux des collèges "répulsifs » accueillent des collégiens résidant dans les espaces périurbains est et sud-ouest (Chorges-La Bâtie Neuve / Tallard - La Saulce) dépourvus de collèges.

L'enquête auprès des collégiens a montré qu'un tiers des dérogations sont justifiées par un choix d'options, et un autre tiers pour la recherche de proximité du domicile (et non du lieu de travail). Les autres motifs sont souvent partagés entre les convenances personnelles (reconstitution de la fratrie) et les activités extrascolaires (clubs de sport urbains lié à un niveau de pratique élevé).

\subsection{Difficulté d'accès au collège pour certains élèves du milieu périurbain}

Au regard des comportements spatiaux et du jugement des familles de collégiens scolarisés au sein du bassin de formation de Gap, les conditions de transport pour se rendre au collège apparaissent comme fondamentales. En 


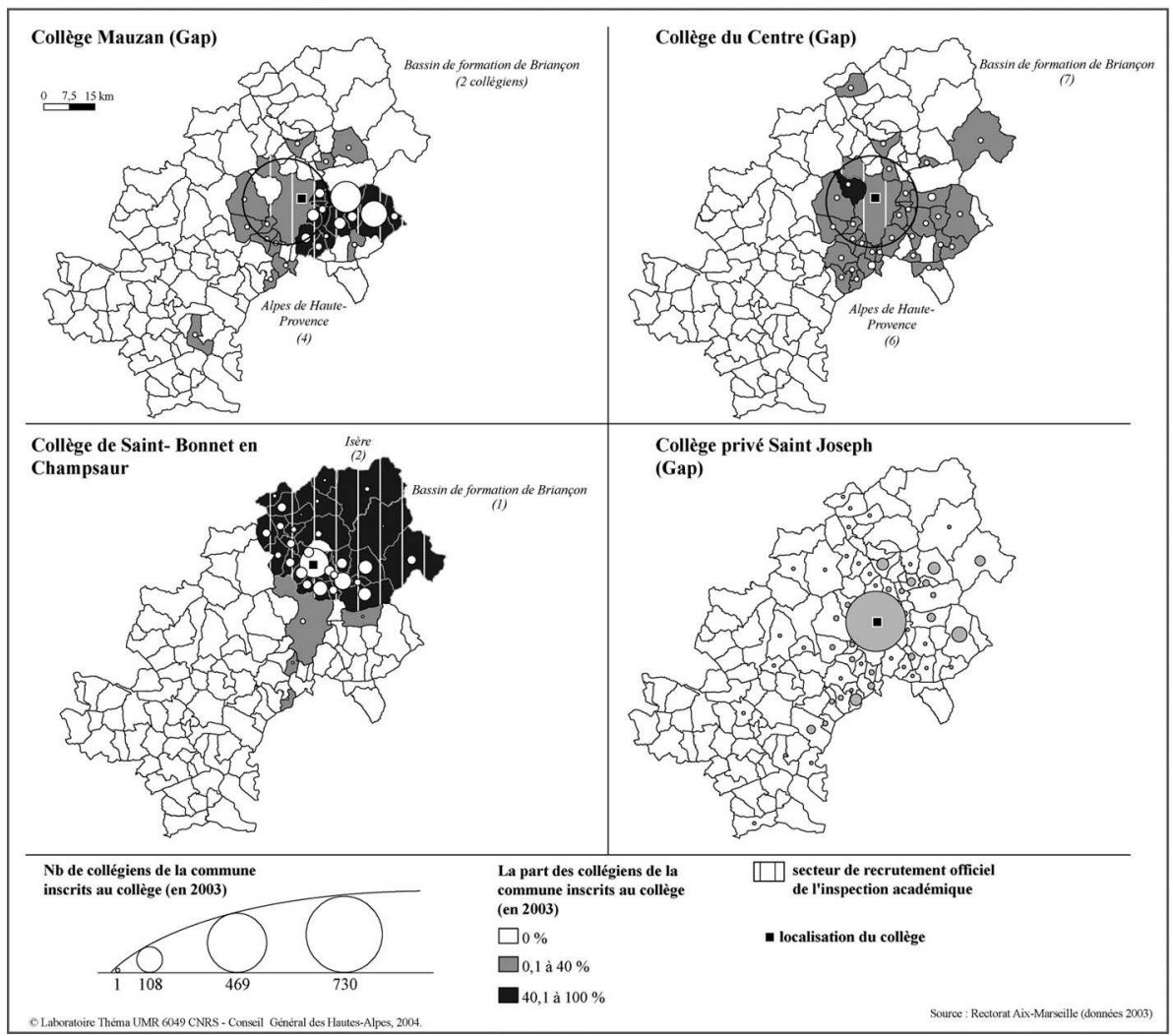

Fig 3 Les aires de recrutement par type de collège.

The recruiting areas in relation to the different kinds of junior high school.

effet, ces conditions de transport justifient très souvent les insatisfactions ou les dérogations à l'affectation imposée par la carte scolaire.

Les distances parcourues ${ }^{10}$ s'avèrent très hétérogènes. En 2003, un collégien de la zone d'étude met en moyenne 6 minutes pour rejoindre son établissement : $71 \%$ d'entre eux sont situés à moins de 10 minutes et $7 \%$ à plus de 20 minutes. Les collégiens de la zone d'étude mettent légèrement plus de temps que les collégiens français pour se rendre dans leur établissement (moyenne de 4 minutes ${ }^{11}$ ). En terme d'accessibilité, quatre types de collèges peuvent être identifiés. Tout d'abord celui du Centre où $90 \%$ des élèves résident à moins de 10 minutes (élèves essentiellement de Gap). Les collèges Mauzan et de Laragne offrent un profil conforme à la moyenne. Le collège rural de Saint-Bonnet accueille davantage d'élèves résidant à plus de 20 minutes (entre 10 et $16 \%$ ) et moins d'élèves au trajet inférieur à 10 minutes.

10 Conditions optimales de circulation et vitesse maximale autorisée / source mappy.fr /.

11 Source : Ministère Education Nationale, chiffres clés 2002. 


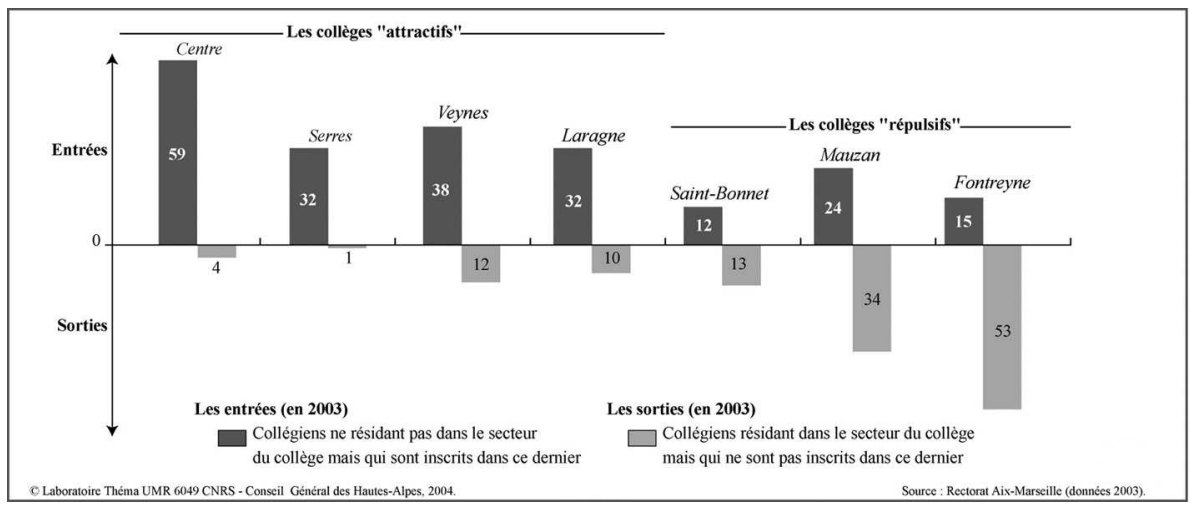

Fig $4 \quad$ Nombre d'entrées et de sorties par collège.

Number of entries and departures for every junior high school.

L'analyse des secteurs de Chorges/La Bâtie Neuve et de Tallard/La Saulce montre que les collégiens de ces deux secteurs affectés sur Gap mettent en moyenne deux fois plus de temps pour se rendre dans leur collège que la moyenne de la zone d'étude (15 et 13 minutes), $10 \%$ seulement d'entre eux étant situés à moins de 10 minutes. C'est donc dans ces deux secteurs, aux temps de parcours allongés par les encombrements aux heures de pointe ${ }^{12}$, que les difficultés d'accès aux collèges sont les plus importantes.

Ces conditions de transport sont donc largement dénoncées puisqu'elles focalisent la majorité des insatisfactions exprimées par les familles de collégiens enquêtées. En effet, $59 \%$ des insatisfaits justifient ce jugement par les difficultés de transport et $20 \%$ par l'éloignement du collège. Ces tendances générales sont encore amplifiées pour les familles résidant sur les secteurs de Chorges/La Bâtie Neuve et de Tallard/La Saulce. Dans ces secteurs, $90 \%$ des insatisfaits justifient ce jugement par les difficultés de transport et l'éloignement. Vu l'importance du facteur transport-accessibilité dans les pratiques spatiales des familles résidant en milieu périurbain, quelle incidence ce facteur peut-il avoir sur la vie au collège et les représentations qu'en ont les parents et les collégiens ?

\section{Le bassin de formation gapencais, un espace vécu}

Afin de cerner l'impact du transport et de l'accessibilité sur les représentations à l'œuvre au sein du bassin de formation de Gap, on privilégie trois angles d'approche : l'offre de formation, la composition sociale et le degré de satisfaction des familles, sorte de ressenti... Dans quelles mesures la

12 Des parcours effectués les 2 et 3/12/2004 ont permis de montrer qu'il faut ajouter environ 20 minutes à ces temps de transport théoriques pour accéder aux collèges de Gap. 


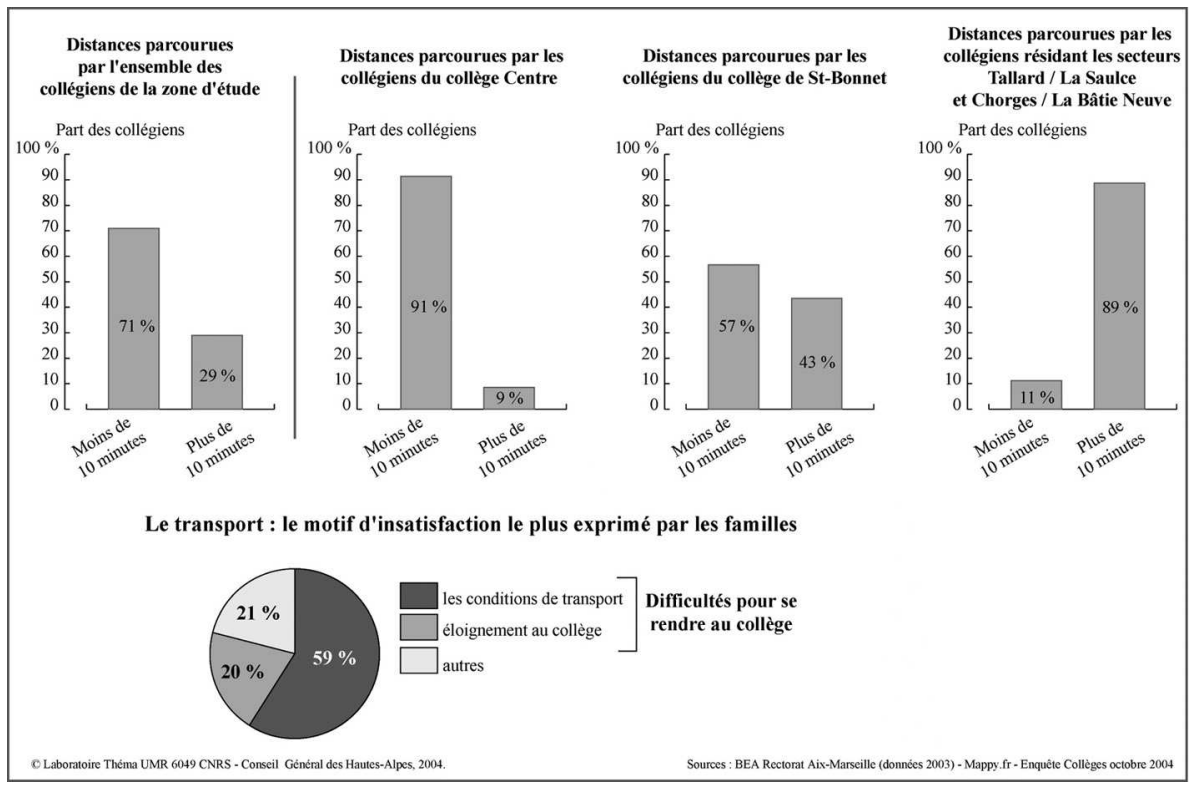

Fig 5 Se rendre dans les collèges du bassin de formation de Gap. Going to junior high school in the educational Gap basin.

recherche de proximité du collège dessine-t-elle les espaces vécus par les parents et les collégiens ?

\subsection{Les options de l'offre de formation des collèges influencent peu les aires de recrutement}

À la rentrée 2003, dix-huit options étaient proposées par les collèges du bassin de formation gapençais. Toutefois, seules quatre options sont présentes dans tous les collèges (anglais LV1, espagnol LV2, italien LV2 et latin). Le nombre d'options proposées par collège varie considérablement en fonction de sa taille $^{13}$. L'offre de formation est donc inégalement répartie sur le territoire selon la taille du collège, même si les options dites de «base » sont présentes dans tous les collèges. La taille d'un collège dépendant directement de son bassin de recrutement, les collèges ruraux proposent très souvent un panel d'options moins large.

Par ailleurs, les enquêtes et les entretiens avec les chefs d'établissement révèlent que moins de $10 \%$ des collégiens suivent une option " originale » (grec ancien, expérimentation chinois, allemand LVl...). Le contingent d'enfants concernés par la recherche de ces options "originales » s'avère donc réduit. À ce titre, seulement $2 \%$ des collégiens ont cité le choix

13 Collège Mauzan : 15 options sur 18 ; collège Centre : 10 options sur $18 \ldots$ collège de Serres : 4 options sur 18 . 
d'option comme raison de satisfaction de leur " affectation collège » actuelle. Une nette différence apparaît donc entre la représentation que développent les acteurs de l'éducation et la réalité. En effet, ces derniers mettent en avant le poids des options dans les choix de dérogations, alors que l'enquête montre que celles-ci guident très peu le choix du collège et n'influencent quasiment pas les jugements positifs comme négatifs sur la vie au collège.

\subsection{Taille des collèges et mixité sociale de leur recrutement}

Très logiquement, l'appartenance sociale des collégiens est calquée sur la répartition des professions et catégories sociales du bassin gapençais, à savoir un tiers de professions libérales, cadres et professions intermédiaires et un tiers d'employés / ouvriers.

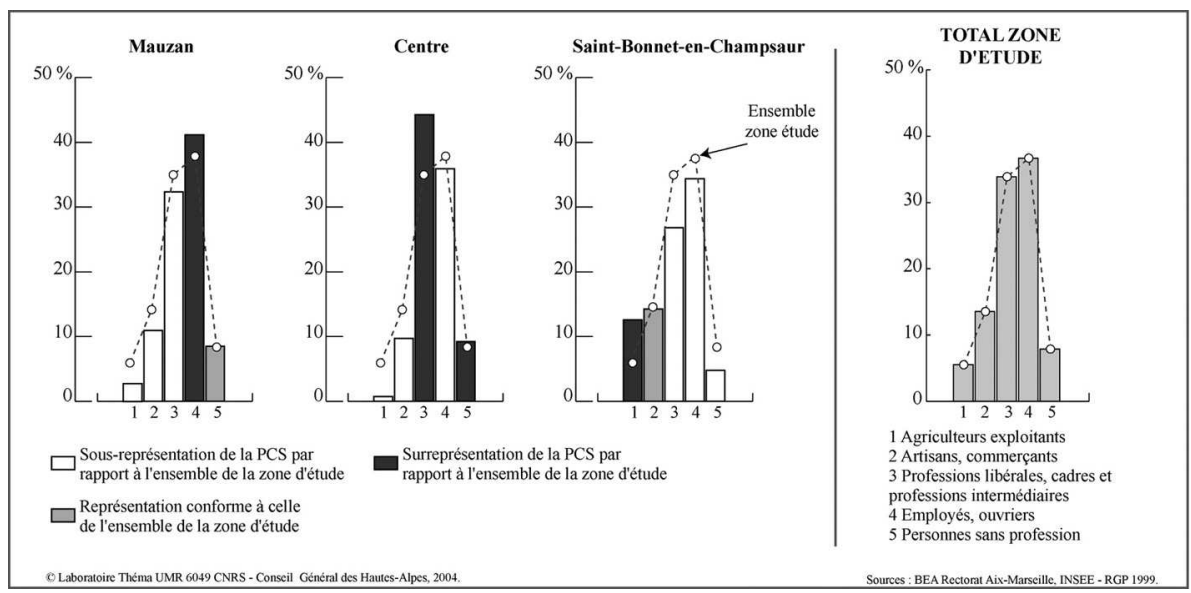

Fig 6 La composition sociale au sein des collèges du bassin de formation de Gap. The social composition within the junior high school educational Gap basin.

Toutefois, les établissements ne présentent pas tous le même profil social, même si «aucun collège ghetto » n'a été identifié. Les collèges ruraux comme celui de Saint-Bonnet comptent moins d'enfants issus des professions libérales, cadres et professions intermédiaires mais plus d'agriculteurs, d'artisans / commerçants. Le collège Centre regroupe davantage d'enfants issus des professions libérales, cadres et professions intermédiaires (classe la plus représentée de ce collège). Les collèges urbains recrutant en périurbain élargi, comme le collège Mauzan, réunissent donc plus d'enfants d'employés / ouvriers.

Au sujet de ces aires de recrutement socio-spatiales, les chefs d'établissement rencontrés insistent sur l'influence de la taille du collège sur les processus de mixité sociale scolaire. Au regard de leurs expériences quotidiennes, un collège de 1000 élèves, tout comme un collège de 300 élèves, ne permettent pas un brassage social optimal. Une trop grande entité favorise la constitution de petits 
groupes aux appartenances sociales semblables, avec un renforcement de cette situation en progressant dans la scolarité. Une trop petite entité, au recrutement spatialement étriqué, court le risque de sur représenter une classe sociale qui vivrait en "vase clos". La taille idéale, pour des échanges sociaux nombreux et variés, est donc fixée à 600 élèves. Il s'agit d'une taille ni trop importante pour garder une «dimension humaine », permettant le contact avec l'enfant, ni trop petite pour risquer la surreprésentation d'une classe socio-spatiale.

\subsection{Un degré de satisfaction élevé, mais fortement dépendant de l'accessibilité et de la taille du collège}

L'interrogation des familles et des élèves sur leur degré de satisfaction les amène à mettre en avant deux logiques structurantes : une petite taille de collège comme élément apprécié et des difficultés de transport et d'accessibilité dénoncées. Seulement $9 \%$ des enquêtés se déclarent peu ou pas satisfaits par leur affectation actuelle (tableau 1). Les satisfactions s'expliquent principalement par la proximité du collège $(80 \%)$, alors que les insatisfactions sont justifiées par les difficultés liées au transport (59\%) et l'éloignement $(20 \%)$. Le degré de satisfaction varie selon les collèges et la commune de résidence. Plus d'un quart des collégiens résidant dans le périurbain est et sud-ouest (Chorges/La Bâtie Neuve et Tallard/La Saulce) se déclarent insatisfaits par leur affectation "collège » actuelle. L'éloignement (plus de $27 \%$ ) et des difficultés (durée) de transport (plus de $60 \%$ ) sont davantage cités par ces élèves périurbains que la moyenne. Les collégiens résidant à Gap sont beaucoup moins nombreux à se déclarer insatisfaits $(4 \%)$. Leur insatisfaction est davantage ciblée sur la trop grande taille du collège $(30 \%)$ que sur les conditions de transport. Enfin, les collèges ruraux des établissements de petite taille regroupent beaucoup moins d'insatisfaits (entre 3 et $4 \%$ pour les collèges de Serres, Saint-Bonnet, Veynes et Laragne).

Au quotidien, le désagrément le plus cité concerne donc les transports (26\% les mentionnent comme un point négatif) et à un degré moindre les conditions de vie scolaire (taille des collèges, ambiance...), alors que les choix d'options demeurent l'élément réunissant le moins de sentiments négatifs. Au final, le facteur essentiel aux yeux des familles, face au territoire scolaire, à sa pratique quotidienne, c'est le transport et l'accessibilité, loin devant les autres éléments. Il s'agit là sans doute aussi du premier facteur de localisation pour le choix de résidence d'un ménage. Par conséquent, la localisation de nouveaux collèges va engendrer à coup sûr une urbanisation de proximité, à cause de l'importance de ce facteur transport. Le collège est donc bien un des équipements les plus structurant dans un bassin de vie.

\subsection{De la réalité... aux représentations : des écarts structurant les logiques d'organisations du bassin de formation de Gap}

Les écarts entre les mesures scientifiques effectuées et les représentations des gestionnaires scolaires sont importants. Les élus ont découvert que la 
Tableau 1 Part des individus enquêtés selon les items abordés et leurs origines géographiques.

The portion of inquired persons according to the chosen criterions and their geographic origins.

\begin{tabular}{|l|r|r|r|r|}
\hline & $\begin{array}{c}\text { Ensemble } \\
\text { Enquêtés } \\
(\mathbf{1} \mathbf{1 0 1})\end{array}$ & $\begin{array}{c}\text { Résidant } \\
\text { à Gap } \\
\mathbf{( 4 8 6 )}\end{array}$ & $\begin{array}{c}\text { Résidant } \\
\text { dans le } \\
\text { périurbain } \\
\mathbf{( 2 3 9 )}\end{array}$ & $\begin{array}{c}\text { Scolarisés } \\
\text { dans les petits } \\
\text { collèges ruraux } \\
\mathbf{( 3 7 0 )}\end{array}$ \\
\hline Indice d'insatisfaction & $\begin{array}{r}9 \% \\
(99)^{*}\end{array}$ & $\begin{array}{r}4 \% \\
(21)\end{array}$ & $\begin{array}{r}27 \% \\
(64)\end{array}$ & $\begin{array}{r}4 \% \\
(15)\end{array}$ \\
\hline Jugements négatifs & $26 \%$ & $22 \%$ & $52 \%$ & $14 \%$ \\
sur le transport & $(286)$ & $(107)$ & $(124)$ & $(52)$ \\
\hline Jugements négatifs & $16 \%$ & $19 \%$ & $23 \%$ & $8 \%$ \\
sur la vie scolaire & $(176)$ & $(92)$ & $(55)$ & $(30)$ \\
\hline Jugements négatifs & $6 \%$ & $4 \%$ & $4 \%$ & $6 \%$ \\
sur les options & $(66)$ & $(21)$ & $(10)$ & $(22)$ \\
\hline
\end{tabular}

Source : enquête UMR Théma du 04 au 08 novembre 2004, 1892 personnes interrogées pour 1101 réponses soit un taux de réponse de $58 \%$.

* 99 enquêtés soit $9 \%$ de l'ensemble $(1101)$.

logique de recrutement du collège privé n'était pas guidée par des convictions car ce collège compte moins d'élèves résidant à l'ouest de gap, où une école privée européenne de 130 enfants est localisée (Tallard), qu'à l'est pourtant historiquement protestant ! La prédominance de la proximité dans les choix et les jugements de vie scolaire au collège, exprimée par les familles constitue également une réelle découverte pour les gestionnaires scolaires. Ces derniers mettaient davantage en avant le recours à des options « originales » ou "d'élite » pour le choix du collège.

Ce constat peut s'expliquer par un morcellement de la gestion scolaire dans laquelle chaque acteur maîtrise une partie des processus analysés :

- l'Inspection Académique gère les crédits éducatifs, les dotations horaires... sans trop considérer les dimensions territoriale et sociale des établissements auxquels elle alloue des moyens de fonctionnement;

- les chefs d'établissements possèdent une connaissance très pointue de l'enfant, au regard de la vie scolaire au sein de l'établissement qu'il gère quotidiennement, sans avoir toujours une vue d'ensemble du territoire (physique, économique et social) et des contraintes budgétaires de l'Éducation Nationale ;

- les collectivités territoriales, et notamment les conseils généraux, intègrent la gestion des collèges dans une vision d'aménagement du territoire sans maîtriser directement l'intérêt de l'enfant et les réalités budgétaires de l’Éducation Nationale.

Chaque partie formant le système scolaire ignore une face du problème dans la mesure où ses compétences ne couvrent pas l'intégralité des pro- 
cessus traités. Le concept de territoire scolaire est donc à même de créer une interface commune à tous les acteurs concernés. À ce titre, le transfert de compétences aux collectivités locales doit permettre "d'injecter du territoire » dans les politiques et stratégies scolaires. Mais tout cela doit être mesuré et réalisé de manière collective ${ }^{14}$ afin de ne pas aboutir à une situation inverse débouchant sur une discrimination territoriale devant l'offre éducative avec des espaces suréquipés et d'autres dépourvus.

\section{Conclusion}

L'étude du bassin de formation de Gap constitue un exemple de territorialisation de politique publique éducative en milieu rural montagnard connaissant un dynamisme démographique périurbain. Malgré le caractère spécifique de ce territoire scolaire, il est possible de dégager quelques leçons quant au fonctionnement d'un territoire scolaire périurbain.

Les trois types d'acteurs, dont les décisions sous-tendent ce fonctionnement, considèrent les collèges et le territoire scolaire avec des points de vue radicalement différents. Pour les premiers, les élèves et leurs familles, l'accessibilité des collèges dans le territoire scolaire compte avant tout. Pour les seconds, acteurs de l'Éducation nationale, le respect de la réglementation / législation nationale (les normes en vigueur) est premier. Par ailleurs, cette catégorie d'acteurs porte une grande attention au coût et à l'efficacité des mesures envisagées. Enfin, les élus des collectivités territoriales, troisième type d'acteurs, se focalisent en premier sur l'égalité, l'équité des citoyens face au service public, ainsi que sur le développement durable.

L'absence de territorialisation de la politique scolaire abouti à l'absence récurrente de liaison entre le maillage complexe de l'éducation nationale et les réalités territoriales. Les nouvelles compétences des collectivités locales (gestion de la carte scolaire des collèges confiée aux conseils généraux), peuvent permettre d'atténuer ce constat. Il faudra sans doute de nombreuses années à ces collectivités territoriales pour s'approprier pleinement ce type de dossiers, à l'instar des conseils régionaux vis à vis de la planification de l'offre de formation des lycées professionnels et techniques. Ces transferts de compétence peuvent permettre de dépasser la simple élaboration de la carte scolaire pour créer des territoires collégiens qui se caractérisent par des mises en réseau et une coordination efficace des déplacements des élèves. Il convient donc d'étudier la dimension, la nature et l'identité du territoire scolaire collégiens à créer, bien plus que la localisation précise de tel ou tel établissement.

Le choix de création et de localisation de nouveaux collèges situés en périurbain, peut être pleinement justifié par la mise en avant des apports

14 Le Conseil Général des Hautes-Alpes a par exemple présenté et soumis à un vote consultatif son projet au CDEN (syndicats de professeurs et parents d'élèves, chefs d'établissements, élus, personnel TOS...). 
réciproques de chacun des éléments du couple "collège - territoire ». Il importe donc de donner un rôle extrascolaire important au collège périurbain, en complément de son rôle scolaire, afin de multiplier les apports sociaux, culturels, sportifs (exemple du collège siège d'une association, lieu de spectacle occasionnel, etc.)... au sein de ces espaces bien souvent dépourvus de services à la population. Le profil d'un collège périurbain est donc radicalement différent de celui du collège urbain. Ainsi, ce collège s'intègre dans son territoire et acquiert une légitimité socio-spatiale qui en fait un équipement collectif à forte identité locale.

Les processus analysés ici concernent les collèges en France métropolitaine, établissements-équipements structurant de proximité par excellence, mais qu'en est-il, à un niveau d'échelle régionale, des lycées ? La recherche de proximité spatiale dans le choix de l'établissement y est-elle aussi affirmée ? Le poids des options donc du niveau scolaire y est-il plus important? Par ailleurs obtiendrait-on les mêmes résultats au sujet de collèges périurbains d'Outre-mer, soumis eux aussi à une forte croissance démographique ? Rien n'est moins sûr.

\author{
Caro P. \\ Laboratoire ThéMA, Université de Franche-Comté \\ 32, rue Mégevand - 25030 Besançon Cedex \\ 03.81.66.54.29 - patrice.caro@univ-fcomte.fr
}

\title{
Bibliographie
}

Caro P., Faivre E., Grosjean F. (2004), «Étude stratégique pour la localisation de collèges sur le bassin gapençais », étude pour le Conseil Général des Hautes-Alpes.

Francois J.C. (1998), Discontinuités territoriales et mise en évidence de systèmes spatiaux dans l'espace des collèges de l'agglomération parisienne in L'espace géographique $\mathrm{n}^{\circ} 1$.

Giband D., Holz J-M. (2001), Constructions scolaires et reconstruction du territoire dans un departement en mutation démographique, I'exemple de la plaine du Roussillon. Populations, espace et sociétés ? REM ? vol 49, n²196, pp. 305-328.

Moracchini C. (1992), Système éducatif et espaces fragiles, les collèges dans les montagnes d'Auvergne. Clermont-Ferrand, Université Blaise Pascal, CERAMAC, 255 p.

Observatoire Régional des Métiers (ORM), (2002), La zone d'emploi de Gap. in outils d'analyse de la formation et de l'emploi en PACA, 22 pages.

Poirey J.-L. et al. (2001), « L'enseignement scolaire dans les milieux ruraux et montagnards. Tome 1, espaces ruraux et réussites scolaires ", Observatoire national de l'école rurale, Annales littéraires de l'Université de Franche-Comté, 124 p.

Poirey J.-L. et al. (2003), « L'enseignement scolaire dans les milieux ruraux et montagnards. Tome 2, au seuil du collège ", Observatoire national de l'école rurale, Annales littéraires de l'Université de Franche-Comté, 124 p.

Sittler M. (1998), La représentation de leur espace de formation après le collège par les élèves de troisième du Pays de Montbéliard. Université de Franche-Comté, Mémoire de DEA de Géographie, 68 p. 